\title{
Development of a Roll-in Oriented Machine for Maize
}

\section{Shelling}

\author{
Kareem Buliaminu \\ Department of Production Engineering, University of Benin, Benin City, Nigeria
}

Received: April 26, 2011 / Accepted: May 09, 2011 / Published: September 25, 2011.

\begin{abstract}
Maize is one of the most important cereal crops in Africa, and Nigeria in particular. It is useful as raw material in many food industries. Traditionally, maize is shelled using manual methods and the yield is always meager. The use of shelling machine can improve the yield tremendously. The problem of the existing shelling machines is in the area of increased damage to the grains while shelling them from the cobs. This made the manual method better than the machining method because of low grain damage witnessed in the latter. Therefore, an effort towards minimizing grain damage during shelling process will be commendable. A shell machine with a roll-in oriented mechanism is proposed for achieving this objective. A maize shelling machine of $8 \mathrm{~kg}$ per minute capacity was developed. This capacity formed the bases of designing for shelling drum, shaft, rotating cylinder (roll-in mechanism), feed rate, torque on shaft, and power required to run the machine. The performance evaluation of the developed machine showed an efficiency of $88.8 \%$ over the manual shelling. This is an improvement over the existing shelling machines whose efficiency ranging from 50\%-65\% as compared to the manual method.
\end{abstract}

Key words: Roll-in mechanism, shelling, manual, maize, performance evaluation.

\section{Introduction}

Maize is one of the most important cereal crops in Africa, and Nigeria in particular. Maize is known botanically as zea may, and as corn. Maize varieties covered dent maize, sweet maize, popcorn, flour corn, and waxy corn. It is useful as raw material in the food industries. A large proportion of processed corn is consumed fresh. The production of maize is quick, easy, cheap, and economical as compared with other food crops including palm tree, and cocoa, which may take several months for maturity. Processed corn is used in the manufacturing of many products, ranging from breakfast foods, corn meal-flour and grits, starch, corn syrup, corn oil, spirits, acetone, chemical, absorbent, seed, and silage [1]. Therefore, the large quantity of maize is needed to meet the need of the agro-allied industries in providing the afore-stated items for the

Corresponding author: Kareem Buliaminu, Ph.D., senior lecturer, research fields: industrial systems innovation and maintenance. E-mail: karbil2002@yahoo.com, bkareem@futa.edu.ng. use and survival of humanity. An increased quality of maize can be enhanced by devising an effective method of processing. The sequence of maize processing includes, harvesting, de-husking, shelling, winnowing, drying, bagging, and storage. Traditionally, maize is shelled using manual methods and the yield is always meager, and cannot meet the large demand of maize by the agro-allied industries [2]. In order to meet up with the demand of local farmers and the industries, using maize as their major raw material, a mechanized system should be devised for easy stripping of grains from the cob by rubbing or beating [2], called shelling. Maize shelling is defined as removal of grains from the cobs by the initial impact, and rubbing action as the material passes through a restricted clearance between the cylinder, and concave bars [3]. The use of shelling machine can improve the yield tremendously.

In literates, many shelling machines have been developed: hand operated, power (mechanical) operated, and tractor operated maize shelling machines are examples. Many types of hand operated maize 
shelling machine are in existence; these include duplex, husking hooks, and pins division corn shelling machines [3]. The performance of these hand operated shelling machines is limited, and can cater for only domestic maize requirement. Power operated machines included mohinder, radam, amar, sherdur, amude, spike tooth tropical motorized maize shelling machine, and francis smith shelling machine have been used till date, and can handle large amount of cobs [4, 5], but the cost of purchase is prohibitive for the local farmers. Tractor operated shelling machine is the most costly among maize shelling machines, because it demands additional cost of tractor for the operation of the machine; but has an added advantage of providing facility for cobs transportation. The high cost of tractor operated maize sheller, has made it unbearable for the use of peasant farmers for their maize processing, instead, manually operated machines were used with is time consuming and requires high energy demand [4].

Apart from high cost of powered-maize-shelling machines procurement, the existing shelling machines have another problem in the area of increased damage to the grains while shelling them from the cobs. This made the manual method better than the machining method because of low grain damage witnessed in it. Therefore, an effort towards minimizing grain damage while engaging in mass production, during shelling process, will be commendable. Many literates have identified some factors that affect the effective shelling of maize in machines; and have given appropriate proportion of them that would enhance faster shelling with less damage to the grains. These are grain moisture, rate of feeding, cylinder speed, shape of shelling chamber, cylinder concave clearance, and corn-ear orientation [6, 7]. Among these factors corn-ear orientation is found to have highest influence on the mechanical damage of the corn grains. Therefore, information on the most influencing factor is needed by designers, in order to incorporate the right shelling cylinder into the shelling machine that will promote high efficiency with few corn grains damages.

In literature, however, three methods of orientation of corn-ear into the shelling chamber were exhaustively debated [7]. These are: roll-in orientation, where the cob is fed with its axis parallel to the cylinder axis; tip-in orientation, where the axis of the cob is perpendicular to the cylinder axis; and random orientation, where the cob is thrown or fed randomly into the cylinder. From the extensive deliberation from the past studies on these cob-orientations [5-7], the conclusion was drawn that roll-in orientation produced the least damage for all moisture contents of maize grains tested, while the tip-in, suffered the most damage, and the random, experienced medium level of damage. Hence, a roll-in orientation mechanism is a feasible mechanism for damage reduction in corn grains during shelling operations. This has motivated the development of roll-in oriented machine for maize shelling.

\section{Roll-in Oriented Maize Shelling Machine Development}

Design, fabrication, and performance testing were carried out in developing the roll-in oriented maize shelling machine.

\subsection{Design Calculation}

In designing for various parts of the machine, capacity of the roll-in shelling chamber was determined first. This was done by estimating average weights (mass), $m_{g}$, and diameters, $D_{g}$, of the randomly selected 10 samples of maize cobs, of different orientations, unshelled; then after hand shelling. From this, differences between the weights, and diameters, before and after shelling, were the bases on which the average grain weight, and diameter were estimated, respectively. The outcomes from average grain weights and diameters were used to determine appropriate clearance between the machine casing and the rotating (roll-in) cylinder. Then the capacity was estimated based on the size and configuration of the casing and 
roll-in mechanism, as well. The details of the design configuration of the casing and roll-in cylinder are shown by Fig. 1; where, $S$, is the length of the hexagonal spike, $X$, the distance between the spikes, $d$, $L_{c}$, the diameter, length, of the roll-in cylinder, $D$, the diameter of the casing, $C$, spike height, $L s$, the length of shaft in the shelling chamber, and $L$, is the length of casing. At the stated specifications of $S=15 \mathrm{~mm}, X=$ $30 \mathrm{~mm}, d=166 \mathrm{~mm}, \mathrm{Ls}=445 \mathrm{~mm}, D=216 \mathrm{~mm}, C=$ $15 \mathrm{~mm}, L s=55 \mathrm{~mm}$ and $L=615 \mathrm{~mm}$, the shelling drum capacity of $0.11686 \mathrm{~m}^{2} \mathrm{~m}$ was obtained based on difference between surface area of the casing, and the roll-in cylinder, which were estimated from the relations $\Pi D^{2} / 2+\Pi D L$, and $\Pi d^{2} / 2+$ $\Pi d L_{c}$, respectively. From the analysis of the data obtained from the measurements of the cob length, $L_{m}$, cob diameter before shelling, $D_{1}$, cob diameter after shelling, $D_{2}$, grain diameter, $D_{g}$ and grain (cob) weight (mass) for the 10 maize cobs samples, an average diameter of $10 \mathrm{~mm}$ was estimated for the grains, whereas an average mass of $0.21 \mathrm{~kg}$ was obtained for the cobs. Based on this, a concave hole diameter on the drum must be greater than $10 \mathrm{~mm}$ for easy dropping of maize grain after shelling; therefore concave or vein hole diameter of $14 \mathrm{~mm}$ was used. The clearance between the casing and the roll-in cylinders, which is the average difference of their diameters, was estimated as $25 \mathrm{~mm}$. The mass of the hexagonal nut ( $\mathrm{M}$ 19), $m_{h}$ used as spites (38 in Nos.) was computed from $\ell v$; where, $\ell$, is the density of mild steel, 7,820 $\mathrm{kg} / \mathrm{m}^{3}$, and $v$, the volume; as $12.6 \mathrm{~kg}$. Similarly, a mass, $21.1 \mathrm{~kg}$ was estimated for the 3 rectangular spikes on the roll-in mechanism, making a total mass of $33.7 \mathrm{~kg}$. Using similar relation (with density of cast iron, $7,200 \mathrm{~kg} / \mathrm{m}^{3}$ ) afore-stated the mass (weight) of the shaft, roll-in cylinder, the spites, and their combination, were estimated as $2.346 \mathrm{~kg}$ ( $w, 23.5 \mathrm{~N}, g$, acceleration to gravity, =10 was assumed), $\mathrm{W}_{1}, 23.46 \mathrm{~N}, 46.3 \mathrm{~N}$, and $W_{2} 69.8 \mathrm{~N}$, respectively. These weights were acting on the shaft, $W$, with pulley weight, $W_{3}, 15 \mathrm{~N}$. Using principle of moment, reactions at the two supports, $R_{1}$ and $R_{2}$ was estimated as $40.2 \mathrm{~N}$, and $68.1 \mathrm{~N}$, respectively. The maximum bending moment on the shaft was estimated as $111.98 \mathrm{Nm}[8,9]$. The velocity of the roll-in cylinder was computed from the ratio of the displacement of a cob (that is, $0.615 \mathrm{~m}$ ) and the time taken to make the displacement. The maximum number of cobs the drum capacity can process in a second was estimated as 4 ; therefore a cob took $0.25 \mathrm{~s}$ to process. Hence, the velocity is $2.46 \mathrm{~m} / \mathrm{s}$. By using appropriate relations, angular velocity $(\mathrm{rad} / \mathrm{s})$, speed (rev/min), centrifugal force, $F_{N}$, force of shelling (N), taking friction into consideration, and total forces in the shelling chamber, was estimated. The torque, $\tau$, developed by the system was estimated as $36.89 \mathrm{Nm}$ from, $f . r$, where, $f$, is the total forces in the shelling chamber, and $r$, the radius of the rotating member. Therefore, the power $P$ of 1,409 Watt $(\approx 2$ Hp) was obtained for the roll-in mechanism using the relation, $\tau W$, where, $W$, is the angular velocity of cobs (rad/s) [10-14]. Hence, electric motor of $2 \mathrm{Hp}$ was selected for the rotating members, for the effective shelling of the maize grains. Other parameters estimated are presented in Table 1.

\subsection{Fabrication}

In fabrication, mild steel was used for the fabrication of the casing, roll-in cylinder, spikes, frame, hopper, and platform. Nickel Chromium alloy steel or cast iron are good as shaft material because of its ability to withstand torsion. A roller-contact bearing was selected because it enhances shaft alignment, easy replacement, lubrication, and can withstand torsion and bending loads. V-belt was selected due to its simplicity, low maintenance cost, ability to absorb shocks and vibration. The welding was done using oxyacetylene gas welding equipment, and the major machining processes carried out comprise bending, cutting, turning, drilling, and boring operations.

The frame is the part on which the machine is mounted. It was constructed from $50 \mathrm{~mm}$ by 50 $\mathrm{mm}$ mild steel angle iron. The roll-in cylinder of the 


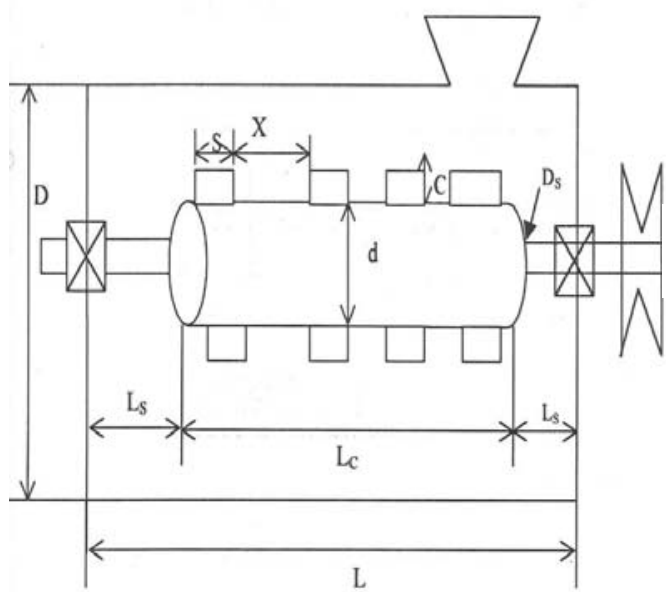

(a)

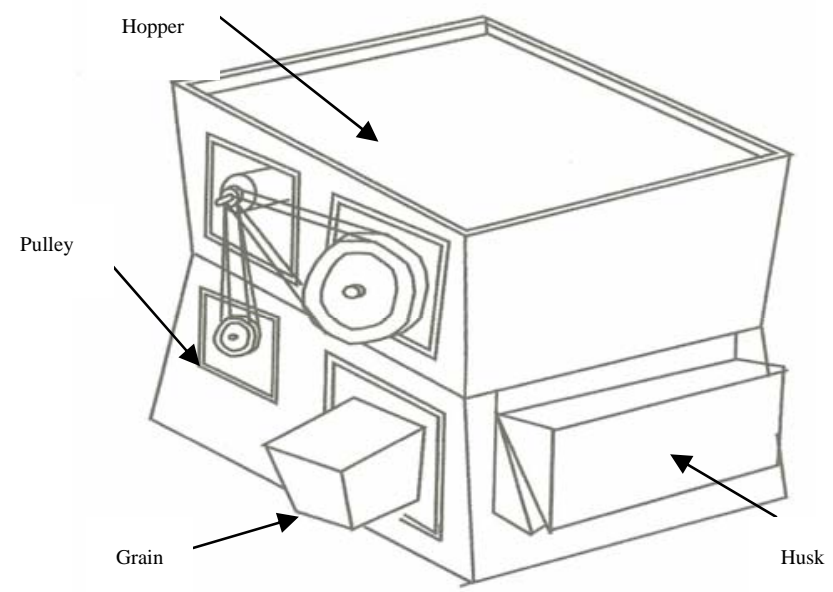

(b)

Fig. 1 The roll-in maize shelling machine. (a) Design configuration, (b) Assembly.

Table 1 Results of the design parameters.

\begin{tabular}{lll}
\hline Components & Parameters & value \\
\hline Shaft & Maximum shear stress & $22.7 \times 10^{4} \mathrm{~N} / \mathrm{m}^{2}$ \\
& Angle of twist & $0.001^{0}$ \\
& Critical speed & $2,500 \mathrm{rev} / \mathrm{min}$ \\
Blower/motor Pulley & Speed & $500 / 2,500 \mathrm{rev} / \mathrm{min}$ \\
Roll-in/motor Pulley & & $250 / 2,500 \mathrm{rev} / \mathrm{min}$ \\
Roll-in/Blower & Centre distance & $210 / 210 \mathrm{~mm}$ \\
Roll-in/Blower & Width & $50 / 25 \mathrm{~mm}$ \\
Belts & Belt lengths & $415 / 415 \mathrm{~mm}$ \\
\hline
\end{tabular}

shelling machine is mounted on bearings (roller-contact bearing) on both ends of the frame. The frame also carried an attachment, which carries an electric motor that drives the roll-in mechanism, and damper, which reduces vibration while in operation. The hopper formed the feeding chute through which unshelled maize cobs are fed into the shelling chamber. The cobs fell under gravity into the chamber. The throat of the hopper is made narrow to prevent the machine from being choke-fed, since the maize cob needs to posses kinetic engorge before entering the shelling chamber. The topmost part of the hopper measured 250-350 mm, while the lower part, which led to the chamber was ranged $200-240 \mathrm{~mm}$. The platform provided at the top of the hopper is meant to assist the capacity of the hopper, and the cobs are packed on it, and gradually fed to the machine; this reduces the labour required to operate the machine. The shelling chamber comprises the shelling drum, cylinder, and the concave (vein). The roll-in cylinder is the main shelling mechanism of the machine. It is situated near the top of the machine and carried on the shaft, which runs the cylinder from the pulley powered by electric motor. The spikes are welded perpendicularly to the axis of the drum, and arranged in angular form. The essence of such arrangement is to make the spikes act as beaters and conveyor as well, moving the waste cobs to its exit.

The concave is the lower part of the shelling chamber; it comprises $3 \mathrm{~mm}$ thick mild steel plate of length $470 \mathrm{~mm}$, which rolled into circular shape of 216 $\mathrm{mm}$ diameter, with holes of about $11.5 \mathrm{~mm}$ diameter. The concave assisted the rotating cylinder to beat the grains out of the cobs by striking the ears against it, and served as separator, as well. It separates detached grains from the cobs. The clean grain outlet is situated 
across the blowing chamber to allow the air stream to blow the dust through its exit. The grain outlet is made of mild steel sheet of 200 by $200 \mathrm{~mm}$ folded to a rectangular section. The husk outlet situated on the opposite end of the shelling drum. The husks are conveniently discharged at the exit, and the grains are removed by the vibration of the machine; and the few grains accompanied the husks could easily find their way back to their exit through perforated end of the exit. The blower chamber comprised the blower (shaft on which blades are arranged serially to give a blower of the centrifugal flow type). Mild steel sheets, 100 by $425 \mathrm{~mm}$ are mounted on the blower shaft, serially, to a blow of air stream as it rotates. They were welded on the shaft, with a sheet of 900 by $455 \mathrm{~mm}$ mild steel folded to give the cylinder a casing, forming the blower casing. The assembled roll-in oriented maize shelling machine is shown in Fig. 1b. The production cost of the machine is about forty four thousand Naira (N44, 000), excluding the cost of labour (Table 2). This is not too expensive for the low income farmers of the developing countries including Nigeria.

\subsection{Performance Evaluation}

Performance evaluation of the machine was carried out using ripped and sterilized palm fruits. The harvested ripped fruit was boiled for two hours for complete sterility. The sterilized fruit was processed in batches, stating from $6 \mathrm{~kg}$ batches, then other batches with cumulative increase of $4 \mathrm{~kg}$, up to a maximum of $26 \mathrm{~kg}$.

\section{Results and Discussion}

The coefficient of performance of the maize Sheller developed was evaluated in terms of the fraction of the maize grains shelled after feeding the hopper with few maize cobs, and then loading the hopper to fill its maximum capacity, in sequence. The results obtained when maize cobs of different weights are fed into the machine are presented in Table 3. From this results shelling efficiency, which is the percentage of the ratio between weight of the shelled grain and the expected weight of grains, was estimated as $88.8 \%$, whereas the operational shelling capacity, which is the ratio of the mass of the shelled grains and the time taken to make

Table 2 Estimate of the cost of materials.

\begin{tabular}{llccc}
\hline S/N & Description & Quantity & Unit price (N) & Total price (N) \\
\hline 1 & Bearings & 4 & 1,000 & 4,000 \\
2 & Bolt and nuts & 44 & 20 & 880 \\
3 & V-belt & 2 & 300 & 600 \\
3 & Angle Iron, std. & 2 & 3,000 & 6,000 \\
4 & Mild steel sheet std. & 3 & 4,000 & 12,000 \\
5 & Pulley & 3 & 2,500 & 7,500 \\
6 & Transmission shaft (shelling \& blower) & 2 & 5,000 & 10,000 \\
7 & Mild steel rods (20 mm, 35 mm) & 2 & 1,000 & 2,000 \\
8 & Hacksaw blades and other consumables & & 2,000 \\
\hline
\end{tabular}

Table 3 Performance results for varying mass of maize cobs shelling.

\begin{tabular}{llllll}
\hline Unshelled cobs (kg) & Shelled cobs (kg) & Time taken (min.) & Cobs weight (kg) & $\begin{array}{l}\text { Expected grains' } \\
\text { weight }(\mathrm{kg})\end{array}$ & $\begin{array}{l}\text { Unshelled corn and } \\
\text { the losses }(\mathrm{kg})\end{array}$ \\
\hline 100 & 80 & 10 & 10 & 90 & 10 \\
200 & 20 & 22 & 178 & 15 \\
300 & 175 & 40 & 55 & 255 & 28 \\
400 & 260 & 40 & 60 & 342 & 35 \\
500 & 360 & 50 & 440 & 40 \\
\hline
\end{tabular}

Average mass of shelled maize $=80 \mathrm{~kg}$

Expected mass of maize grain $=90 \mathrm{~kg}$

Efficiency $=88.8 \%$ 
the shelling, was established to be $504 \mathrm{~kg} / \mathrm{hr}$.

The shelling efficiency of the machine is high enough to guaranty effective delivering of maize grains for the satisfaction of the domestic and industrial demands. It was also discovered from the results that the shelling rate is high when the cobs are fed to hopper gradually than if stocked amassed. The rate of shelling was high at the beginning, and steadily reduced as time increases further. The grains' loss is increased with time and quantity of unshelled cobs fed into the hopper. In all cases the loss due to damage and unshelled was less than $10 \%$. This is an improvement over the existing machines with efficiency ranging from $50 \%$ to $65 \%$ [1-3].

\section{Conclusions}

The developed roll-in fed maize shelling machine has solved the problem of major damages on grains suffered from the existing shelling machines involving tip-in, and random methods of feeding. The cost of machine is moderate and affordable by low income farmers. The machine has a processing capacity, which is enough to take care of domestic and industrial consumers. Electrical motor generated sufficient power to run the entire machine elements. Mild steel was used largely for the fabrication of the body, and the transmission shafts are made from high grade steel. The blades on the blower shaft are made of mild steel, for effective blowing of the husk from the grains, and the grains can be bagged directly as it is coming from the machine, as well. The noise (vibration) is reduced considerably as the bearings, absorbers (dampers), and frame provided balanced alignment. The efficiency of the machine is satisfactory and provided a reduced proportion of grains' damage. Finally, the cost of the machine could be reduced further if mass produced.

\section{References}

[1] T.A. Adebusuyi, Design and Construction of a Maize Sheller, Institute of Agric. Res. and Training, Ibadan, 1983.

[2] B.A. Adewumi, T. Adegbulugbe, Design of disc type sheller, T. Science/Engineering Tech. 91 (1991) 19.

[3] M.O. Ayetigbo, Design and construction of maize sheller, PGD Thesis, Federal University of Technology, Akure, Nigeria, 2001,

[4] K. Baikstron, Latest in field shelling of corn, ASAE Journal 9 (118) (1964) 484.

[5] B. Fagoroye, Evaluation of some parameters associated with mechanical shelling of maize, HND Project, IART, Ibadan, Nigeria, 1995.

[6] Food and Agricultural Organisation (FAO), Post Harvesting and Year Processing Technology of Africa Staple Foods, FAO-Bulletin-A Technical Compendium, 1991, p. 25.

[7] P. Galnze, Mechanised Production of Maize in Tropical and Sub-tropical Regions, Technical Fundamentals, Leipziz Publication, 1972, pp. 136-155.

[8] A. Hall, M.S. Holowanko, H.G. Laughlin, Machine Design, Schaum's Outline Series, McGraw-Hill, New York, 1988.

[9] H.A. Harold, Mechanical Design Systems, McGraw-Hill, London, 2003.

[10] R.M. Lien, C.G. Hanh, Effect of field shelling on popcorn quality, ASAE Journal 18 (5) (1995) 856-858.

[11] K. Napgal, Machine Design, Khana Publishers, New Delhi, 1984, pp. 516.

[12] G.H. Ryder, Strength of Material, 4th ed., Macmillan Press Limited, London, 1954.

[13] J.E. Shigley, Mechanical Engineering Design, Mcgraw-Hill, Kogakusha, 1970.

[14] A.A. Stone, H.E. Gulvin, Machine for Power Farming, John Wiley and Sons, pp. 450-451. 